\title{
Association of Arrhythmias in Cardiac Amyloidosis and Cardiac Sarcoidosis
}

\author{
Ibtisam Ashraf ${ }^{1,2}$, Mercedes Maria Peck ${ }^{3}$, Ruchira Maram ${ }^{4,2}$, Alaa Mohamed ${ }^{2,5}$, Diego Ochoa Crespo ${ }^{6}$ \\ 2 , Gurleen Kaur ${ }^{2}$, Bilal Haider Malik ${ }^{2}$ \\ 1. Internal Medicine, Shalamar Institute of Health Sciences, Lahore, PAK 2. Internal Medicine, California Institute of \\ Behavioral Neurosciences and Psychology, Fairfield, USA 3. Internal Medicine, California Institute of Behavorial \\ Neurosciences and Psychology, Fairfield, USA 4. Internal Medicine, Arogyasri Healthcare Trust, Hyderabad, IND 5. \\ Internal Medicine, Memorial Hermann Medical Center, Houston, USA 6. Internal Medicine, Clinica San Martin, \\ Azogues, ECU
}

Corresponding author: Ibtisam Ashraf, ibtisamshrf@gmail.com

\begin{abstract}
Cardiac involvement in amyloidosis and sarcoidosis is poorly understood, and is associated with high morbidity and mortality. Atrial and ventricular arrhythmias, along with conduction defects, are frequent in cardiac amyloidosis and sarcoidosis. Atrial dysfunction in cardiac amyloidosis may result in atrial fibrillation and increases the risk of stroke, making anticoagulation significant and challenging. Ventricular arrhythmia and conduction defects are more common in AL amyloidosis and cardiac sarcoidosis. Premature ventricular contractions (PVCs) from Purkinje fibers trigger ventricular arrhythmias in cardiac amyloidosis, while the inflammation and scarring leading to the reentrant process is the cause in cardiac sarcoidosis. The typical treatment modalities include Class II and III antiarrhythmic drugs and ablation techniques, while corticosteroids and immunosuppressants are indicated in cardiac sarcoidosis to reduce the burden of the disease and arrhythmias. Sudden cardiac death can be a manifestation of both disorders that can be prevented by the Implantable cardioverter-defibrillator (ICD), although the predictive risk factors for primary prevention remain uncertain. In this review, we addressed the current understanding of the pathways involved in inducing arrhythmias in cardiac amyloidosis and sarcoidosis-also, the complications including sudden death and stroke associated with arrhythmia in both diseases. We have discussed other preventive steps needed to minimize arrhythmias to provide symptomatic relief and palliation to patients.
\end{abstract}

Received 07/31/2020 Review began 08/11/2020 Review ended 08/11/2020 Published 08/18/2020

๑) Copyright 2020

Ashraf et al. This is an open access article distributed under the terms of the Creative Commons Attribution License CC-BY 4.0., which permits unrestricted use, distribution, and reproduction in any medium, provided the original author and source are credited.
Categories: Cardiology, Internal Medicine

Keywords: cardiac amyloidosis, cardiac sarcoidosis, arrhythmia, catheter ablation, implantable cardioverterdefibrillator, atrial arrhythmia

\section{Introduction And Background}

Cardiac amyloidosis (CA) and sarcoidosis are typically known as infiltrative cardiomyopathies. The incidence of cardiac amyloidosis is 18 per 100,000 person-years, while cardiac sarcoidosis occurs in $5 \%$ of the patients with systemic sarcoidosis (10-20 per 100,000 individuals) [1,2]. Amyloidosis is characterized by the deposition of insoluble amyloid fibrils in extracellular tissues involving multiple body systems, including cardiac tissue. Several forms of precursor protein significantly affect the heart: light-chain (LC) immunoglobulin, mutant hereditary transthyretin (TTR), wild-type TTR, mutant apolipoprotein AI, amyloid atrial natriuretic peptide localized to the atrium, fibrinogen alpha type and serum amyloid A protein [3]. Sarcoidosis is a granulomatous multisystem disease in which CD4+ T-lymphocytes aggregate to induce a Th1 type immune response [4]. Both diseases may involve cardiac tissue either as a part of the systemic condition or in isolation. Heart failure, conduction abnormalities, and arrhythmias are the most common signs of cardiac involvement [1,4]. Arrhythmias in cardiac amyloidosis vary by amyloidosis type, as conduction defects and supraventricular arrhythmias are more prevalent in transthyretin amyloidosis [5]. Atrioventricular (AV) block is the most common type of arrhythmia in cardiac sarcoidosis, followed by ventricular tachycardia and supraventricular arrhythmia [6].

Cardiac involvement in systemic sarcoidosis and amyloidosis typically results in poor prognosis. They account for around $10-25 \%$ of all sarcoidosis deaths in the US and are generally due to malignant arrhythmias and complete heart block [7]. The precise cause of arrhythmias in CA is less clearly known and is likely to be multifactorial [5]. There is little understanding of the exact mechanism of sudden cardiac arrest and the identification of CA patients that may be eligible for implantable cardioverter-defibrillator (ICD) [8]. Whether implantable cardioverter-defibrillator (ICD) use in such patients prevents sudden cardiac death (SCD) is uncertain. Furthermore, atrial dysfunction in amyloidosis raises the likelihood of intracardiac thrombus formation that can lead to stroke, a probability unknown [9]. Ventricular arrhythmias arising in cardiac sarcoidosis can be fatal and its mechanism and prevalence are not well established. Glucocorticoids are the mainstays of treatment in cardiac sarcoidosis, but data from randomized controlled studies are scarce. Similarly, the precise role of corticosteroids in managing arrhythmias including atrioventricular blocks (AVBs) needs to be established. 
These disorders have diverse clinical manifestations and severe outcomes that render it challenging to diagnose and manage. Also, there is little understanding of electrophysiological abnormalities in infiltrative cardiomyopathies. This review aims to address the cause, relationship, and complications of arrhythmias, based on the most current data, in cardiac amyloidosis and sarcoidosis.

\section{Review}

\section{Pathogenesis of arrhythmias in cardiac amyloidosis and cardiac sarcoidosis}

Cardiac amyloidosis (CA) results from the infiltration of the extracellular spaces by amyloid, isolating, and misshaping the structure of myocardial cells. This deposition increases ventricular wall thickness resulting in restrictive cardiomyopathy [10]. Electrocardiogram (ECG) often shows low voltage QRS complex and pseudo infarct patterns on precordial leads. Several different mechanisms aid myocardial dysfunction. In addition to infiltration, light chains in AL amyloidosis may also induce direct toxicity by increasing the intracellular reactive oxygen species [11]. Evidence of amyloid accumulation is found in perivascular regions and media of intramyocardial coronary vessels that may induce coronary microvascular dysfunction leading to ischemia and hence to myocardial injury [12]. Studies in Sweden, involving cases of familial amyloidosis with polyneuropathy, have specifically demonstrated amyloid infiltration of the sinus node and atrioventricular conduction system $[13,14]$. Consequently, this inflammatory cell injury, structural destruction, and separation of myocytes by amyloid fibrils explain the electrophysiological abnormalities.

Cardiac sarcoidosis (CS) is a granulomatous disease that results in conduction abnormalities and ventricular arrhythmias. The precise cause of sarcoidosis is unclear, however, it seems to affect the basal septum, atrioventricular node (AV node), atrioventricular (His) bundle, the focal regions of the ventricular free walls, and the papillary muscles of the heart [15]. Isolated cardiac sarcoidosis accounts for two-thirds of all cardiac sarcoidosis cases, requiring the diagnosis of CS and the absence of sarcoid involvement of any other organ. Electrocardiograms can exhibit atypical infarction patterns and atrioventricular (AV) blocks, as shown in Table 1 . This cardiac disease typically progresses from localized inflammation to scarring, resulting in atrial and ventricular arrhythmias. Similarly, inflammatory processes tend to include basal interventricular septum and result in atrioventricular blocks (AVB) and bundle branch blocks. In a retrospective study in Finland, atrioventricular blocks were found to be the most frequent presenting symptom in cardiac sarcoidosis, arising in about $44 \%$ of the cases. In contrast, ventricular arrhythmias were the second most common presenting symptom, occurring in $33 \%$ of the cases [6].

\begin{tabular}{|c|c|c|c|c|c|c|}
\hline & $\begin{array}{l}\text { Age at } \\
\text { Presentation }\end{array}$ & $\begin{array}{l}\text { Clinical } \\
\text { Presentation }\end{array}$ & Echocardiography & ECG & CMR LGE & Biopsy \\
\hline $\begin{array}{l}\text { Cardiac } \\
\text { Amyloidosis }\end{array}$ & $\begin{array}{l}6^{\text {th }} \text { or } 7^{\text {th }} \text { decade, } \\
\text { Male }>\text { Female, } \\
\text { ATTR variant } \\
\text { common in African } \\
\text { American }\end{array}$ & $\begin{array}{l}\text { Heart Failure, } \\
\text { Nephrotic } \\
\text { syndrome, } \\
\text { Peripheral } \\
\text { neuropathy }\end{array}$ & $\begin{array}{l}\text { Increased LV and RV wall } \\
\text { Thickness, Normal or small LV } \\
\text { cavity size, biatrial enlargement, } \\
\text { granular appearance of } \\
\text { myocardium }\end{array}$ & $\begin{array}{l}\text { Low-voltage QRS } \\
\text { complex, Pseudo- } \\
\text { infarct pattern in } \\
\text { precordial leads }\end{array}$ & $\begin{array}{l}\text { Global } \\
\text { transmural or } \\
\text { subendocardial } \\
\text { LGE }\end{array}$ & $\begin{array}{l}\text { Replacement } \\
\text { of normal } \\
\text { cardiac tissue } \\
\text { by amyloid }\end{array}$ \\
\hline $\begin{array}{l}\text { Cardiac } \\
\text { Sarcoidosis }\end{array}$ & $\begin{array}{l}3^{\text {rd }} \text { or } 4^{\text {th }} \text { decade, } \\
\text { Female }>\text { Male, } \\
\text { More common in } \\
\text { African Americans }\end{array}$ & $\begin{array}{l}\text { Heart Failure, } \\
\text { SCD, CHB }\end{array}$ & $\begin{array}{l}\text { Segmental wall motion } \\
\text { abnormalities and Variable Septal } \\
\text { Thickness }\end{array}$ & $\begin{array}{l}\text { AV } \\
\text { Block/Infrahisian } \\
\text { block, Atypical } \\
\text { Infarction Pattern }\end{array}$ & $\begin{array}{l}\text { Patchy basal } \\
\text { and LV free } \\
\text { wall }\end{array}$ & $\begin{array}{l}\text { Noncaseating } \\
\text { multinucleated } \\
\text { giant cell } \\
\text { granulomas }\end{array}$ \\
\hline $\mathrm{AV}=$ atrioventr & & & $\mathrm{B}=$ complete heart block; LGE = late & gadolinium enhanc & & \\
\hline
\end{tabular}

\section{Atrial arrhythmias}

Atrial fibrillation (AF) and atrial tachycardia (AT) are the most commonly occurring arrhythmias in cardiac amyloidosis. The combination of intramyocardial amyloid accumulation, leading to restricted ventricular relaxation and elevated filling pressures, which eventually causes left atrial enlargement, and the intrinsic left atrial dysfunction due to direct amyloid deposition, are the causes of atrial dysfunction in CA [16]. Atrial fibrillation is more frequent in wild-type transthyretin amyloidosis (ATTRwt) [5]. Barbhaiya et al. performed an atrial arrhythmia substrate analysis, which showed supraventricular tachycardia in all 18 patients with CA [17]. Another study showed that both interventricular septum (IVS) thickness and atrial dimension were associated with an increased rate of supraventricular arrhythmia [18]. Amyloid accumulation in atria isolates atrial myocyte bundles, which is why AF in CA appears to have longer cycle lengths because the mechanism induces a pronounced delay in intra-atrial conduction [5]. 
The precise prevalence of atrial arrhythmias in cardiac sarcoidosis is unclear. Atrial arrhythmias, like AF/AT, are less frequent than ventricular arrhythmias in CS. Viles-Gonzalez et al. documented supraventricular arrhythmia in $32 \%$ of CS cases (atrial fibrillation $18 \%$, atrial tachycardias $7 \%$, atrial flutter $5 \%$, and other types of supraventricular tachycardias 2\%) [19]. In contrast, Cain et al. evaluated 192 patients with extracardiac sarcoidosis (confirmed with a biopsy and CMR imaging) and found that atrial arrhythmias (36\%) were more common than ventricular arrhythmias (27\%) [20]. CS induces inflammation and scarring of the atrial tissue and atrial enlargement due to ventricular dysfunction that leads to atrial arrhythmias. Autopsy of patients with cardiac sarcoidosis showed sarcoidosis lesions in the left ventricular free wall (96\%), the ventricular septum (73\%), the right ventricular wall (46\%), the right atrium (11\%), and the left atrium (7\%) [21]. Multivariate analysis showed that left atrial enlargement was the only independent factor consistent with atrial arrhythmias in CS patients [19].

\section{Atrial thrombus formation in CA}

Cardiac amyloidosis raises the risk of the development of intracardiac thrombus, which can result in cardioembolic stroke. A study showed that $33 \%$ of the 116 autopsy cases had intracardiac thrombi and that the potential cause may be the combination of hypercoagulability, endomyocardial disruption, and endothelial dysfunction due to amyloid deposition [22]. Amyloid accumulation causes atrial mechanical dysfunction that can lead to thrombus formation even in the absence of atrial arrhythmia [9]. In another study, intracardiac thrombus was shown more frequently in AL amyloid than in other forms (35\% vs. $18 \%$ ) [23]. Stroke in CA is not an uncommon complication that can occur even in patients on anticoagulation therapy. Recently, $8.9 \%$ of the 89 patients with CA had an ischemic stroke [24]. Thus, it is crucial to estimate the correct risk of stroke in CA patients with or without atrial fibrillation.

\section{Ventricular arrhythmias}

Ventricular arrhythmias are more frequently observed in light-chain (LC) immunoglobulin (AL) amyloidosis, mainly due to its steep downward path following the onset of heart failure [5]. Dubery et al. examined the 24-hour Holter recordings of 195 patients with AL amyloidosis and found non-sustained ventricular tachycardia (VT) in $27 \%$ of patients [25]. In another study with implanted loop monitors, bradycardia was reported in 20 patients with AL CA death, and there was only one episode of non-sustained ventricular tachycardia, preceded by severe bradycardia [26]. Ventricular fibrillation may be caused by premature ventricular contractions (PVC) originating from the Purkinje system. It can occur in post-MI patients as well as in chronic ischemic cardiomyopathy. A study reported that two CA patients had repetitive VF episodes with each episode preceded by monomorphic PVC [27]. Nevertheless, the precise cause of VF arising in CA is not well understood.

Ventricular tachycardia (VT) is the second most frequent form of arrhythmia in cardiac sarcoidosis. Ventricular arrhythmias in CS are due to reentrant and focal (triggered activity or abnormal automaticity) mechanisms. The scarred areas of ventricular endocardium and epicardium are capable of sustaining a significant number of reentrant circuits and are niduses for re-entry [28]. Naruse et al. reported a sequence of 37 patients with CS in which a total of 57 VTs were induced; 14 VTs were non-sustained, six were polymorphic, six were associated with Purkinje system, and 31 were associated with scar areas [29]. Sustained monomorphic VT in CS is a significant indicator of mortality. It can lead to hemodynamic collapse, which means that these patients need an immediate conversion to sinus rhythm. Also, the risk stratification of ventricular arrhythmias in these patients is necessary.

\section{Conduction defects}

Cardiac involvement in amyloidosis may cause conduction anomalies that are evident to ECG as the narrow QRS complex, bundle branch blocks, fascicular blocks, and atrioventricular blocks (AVB). A recent study has shown that ventricular conduction and repolarization defects are higher in AL relative to ATTR amyloidosis [30]. Conduction abnormalities such as atrioventricular conduction delay are more common than sinus node disease, despite the frequent atrial involvement in CA. Intracardiac electrophysiology (EP) examination of sinus node function in 25 patients with AL amyloidosis showed normal sinus node function in $88 \%$ of the patients tested [31]. On the other hand, a previous study showed that a common morphological abnormality of the conduction system was severe sinoatrial fibrosis [32]. However, patients with cardiac amyloidosis have frequent dysfunction in the His-Purkinje system. It is demonstrated with HV prolongation, which is the conduction time measured from the onset of His potential to the beginning of intracardiac ventricular activation. Reisinger et al. showed that the mean HV interval in 25 patients was $79+/-18 \mathrm{~ms}$ and 23 patients (92\%) had an abnormally prolonged interval $(>55 \mathrm{~ms}$ ) while 12 patients showed a pronounced $\mathrm{HV}$ prolongation ( $>80 \mathrm{~ms}$ ), six of which had an interval $>100 \mathrm{~ms}$ [31]. This suggests a significant infiltration of myocardial tissue, including the conduction system, by amyloid fibrils. Such patients also have a high frequency of sudden death, the HV interval being an independent indicator.

Conduction disorders, including atrioventricular blocks (AVBs), are the most common manifestations of cardiac sarcoidosis, responsible for around half of the cases. The primary cause of this is widespread inflammation and fibrosis in the conduction system, as shown in Figure 1. The right bundle branch block is frequent and can be a symptom of newly developed CS in documented extracardiac sarcoidosis patients. A study showed that $34 \%$ of patients 60 years of age with AVB had CS as an underlying condition [33]. Another 


\section{Cureus}

study found that sudden death was the initial manifestation of sarcoidosis in 10/60 patients (17\%) who died unexpectedly, while complete heart block was the most prevalent conduction disorder in 25 patients, and a complete bundle branch block occurred in 21 patients [21]. Therefore, all patients $<60$ years of age who present with high-degree AV block should be screened for CS.

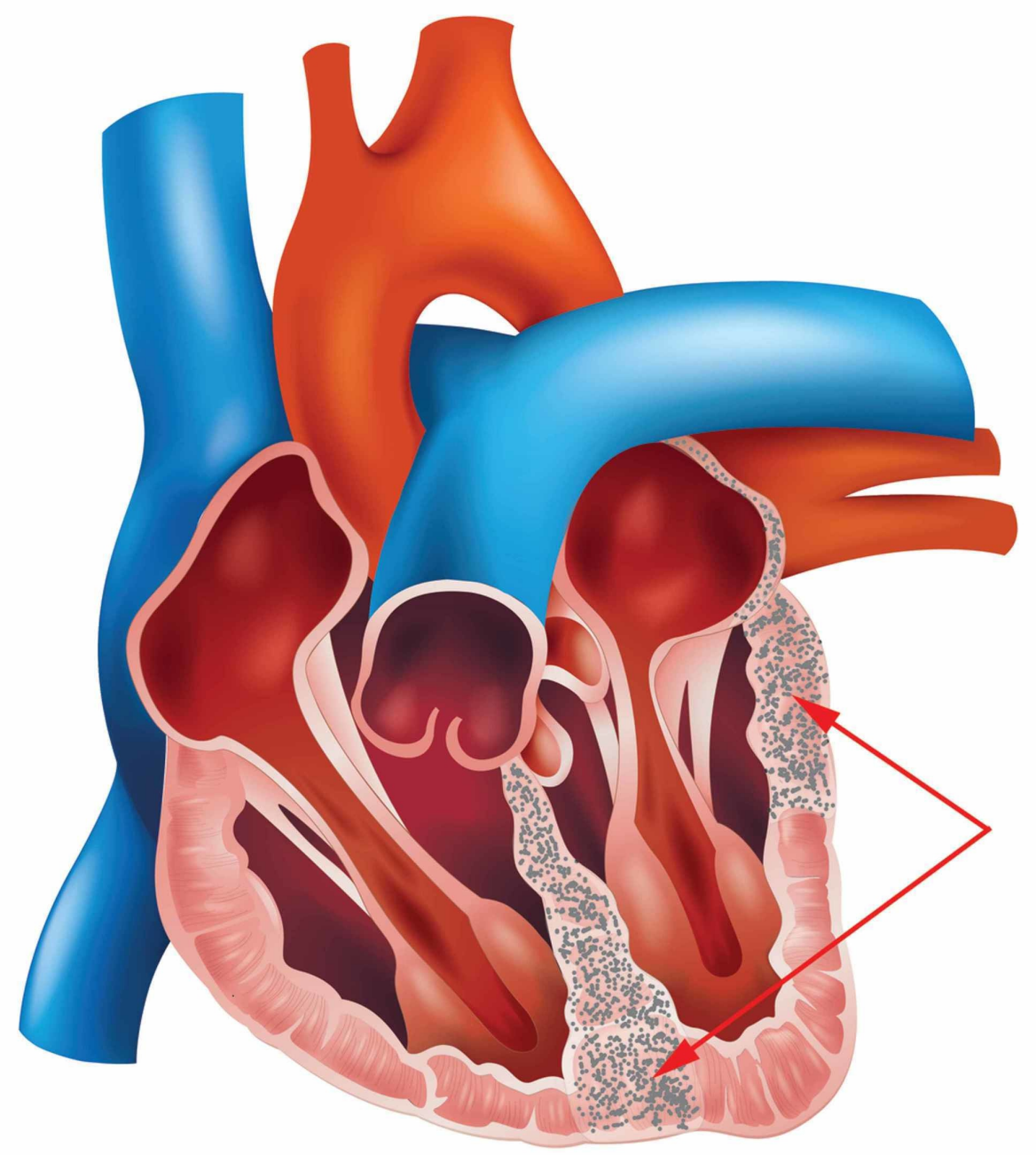

FIGURE 1: Showing Septum and LV fibrosis in sarcoidosis clinically manifested as Heart Block, Heart failure, and Ventricular Tachycardia due to reentrant circuits around the scar.

\section{Management of arrhythmias}

The management of arrhythmias in cardiac amyloidosis and sarcoidosis is a complicated process. The prognosis of both conditions is generally poor, however, antiarrhythmic and immunosuppressive therapy and cardiac ablation techniques are increasingly being used to control the underlying arrhythmias. Implantable cardioverter-defibrillator (ICD) can be used to prevent sudden death due to fatal arrhythmias in these cases.

\section{Antiarrhythmic and Immunosuppressive Therapy}

The evidence on antiarrhythmic medications is limited. Class III antiarrhythmic medications are used to regulate AF/AT in cardiac amyloidosis but are ineffective in cardiac sarcoidosis. Corticosteroid treatment has proven successful in reducing the burden of AF in CS [2]. Anticoagulation is strongly recommended for cardiac amyloidosis patients with atrial fibrillation due to an increased risk of stroke.

Similarly, class III agents are also used to treat ventricular arrhythmias in cardiac sarcoidosis. However, amiodarone can induce pulmonary toxicity in these patients and may be deferred in advanced pulmonary sarcoidosis [2]. Corticosteroid therapy has been effective in treating ventricular arrhythmias and improving 
AV conduction in patients with CS. A study reported a 47\% improvement in AV conduction in patients treated with corticosteroids, with no improvement in the untreated group [34]. Another study showed that the patients who received early treatment with corticosteroids resulted in no ventricular arrhythmia recurrences in 8/11 patients (72\%), and complete recovery of AV conduction in 2/3 patients (67\%) [35]. In addition, methotrexate and azathioprine can be used as an alternative to corticosteroids in these cases.

Ablation

AV node ablation is usually done in CA and CS patients with atrial arrhythmia (AF) and with persistent symptoms despite the optimal medical therapy. It can improve the rate and restore sinus rhythm, providing symptomatic relief in patients with atrial fibrillation. However, there is an increased rate of recurrence in CA patients with AF. A study showed a one-year recurrence rate of $83 \%$ in CA patients compared to $25 \%$ in non-CA patients [5]. Whereas CS patients with AF had a recurrence rate of $22 \%$ ( $2 / 9$ patients) following ablation [36].

Ventricular arrhythmias in CA are caused by PVC arising from the distal Purkinje system. Thus, catheter ablation of the triggering PVC from the Purkinje system can be used as a therapy in these patients. Mlcochova et al. identified two patients with repetitive VF related to cardiac amyloidosis [27]. PVC followed each episode of ventricular arrhythmia, which was not associated with the scar tissue but was drug-resistant. The recurrence of VF was successfully eliminated by catheter ablation.

On the other hand, ventricular arrhythmia in CS is due to a reentrant mechanism and is associated with scar tissue. A systematic review was done involving 83 patients and five studies [37]. The mean age of patients was $50 \pm 8$ years, and the mean ejection fraction was $39.1 \pm 3.1 \%$. The median number of VTs was three per patient, mean cycle length of $360 \mathrm{~ms}$ (326-400 ms). All patients received endocardial ablation, and $18 \%$ required epicardial ablation. From a less strict endpoint (i.e., freedom from arrhythmia or reduction of ventricular arrhythmia burden), 61 (88.4\%) patients improved following ablation. There was a relapse in 45 (54.2\%) patients. Despite the VT recurrence, ablation is quite effective in treating ventricular arrhythmias in CS patients.

Implantable Cardioverter-Defibrillator (ICD)

ICDs could be used as a prophylactic device to prevent fatal arrhythmias leading to sudden death in CA and CS patients. Arrhythmic sudden death is far more common in AL amyloidosis. In a study involving 53 patients with CA who underwent ICD implantation were included [8]. The rate of appropriate ICD shocks was $32 \%$ in the first year and occurred almost exclusively in patients with AL amyloidosis. Appropriate ICD shocks were more frequent in patients with prior sudden cardiac arrest or sustained ventricular arrhythmias. Nevertheless, appropriate ICD therapy did not result in an overall survival benefit. In general, ICDs are not typically given to patients with a life expectancy of less than one year, as is usually the case in cardiac AL amyloidosis, and their application in primary prevention of fatal arrhythmias in CA patients remains uncertain.

ICD implantation is indicated in cardiac sarcoidosis and spontaneous sustained ventricular arrhythmia, particularly those with previous cardiac arrest [2]. According to the 2014 Heart Rhythm Society, class IIb drugs for primary prevention along with implantable cardioverter-defibrillator (ICD) placement are recommended in those patients with mild-to-moderately reduced left ventricular ejection fraction (LVEF 36$49 \%$ ) and/or reduced right ventricular ejection fraction (RVEF <40\%) despite optimal medical therapy and a period of immunosuppression [38]. SCD is not uncommon in CS. The five-year risk of SCD in middle-aged patients is $34 \%$ when the AVB is presented with VT or severe LV dysfunction [2]. So, ICD is also recommended in patients with a permanent pacemaker indication due to atrioventricular blocks (AVBs).

\section{Conclusions}

This article outlines the various arrhythmias that occur in cardiac amyloidosis and sarcoidosis. It is important to understand the association of arrhythmias with infiltrative cardiomyopathies, since fatal arrhythmias are the leading cause of death in these diseases.The precise understanding of the mechanism of atrial and ventricular arrhythmias of cardiac amyloidosis remains uncertain. However, several mechanisms, including inflammatory cell damage, cellular degradation, and separation of myocytes by amyloid fibrils, cause CA arrhythmias. Similarly, inflammation and scarring contribute to arrhythmias in cardiac sarcoidosis. Atrial dysfunction resulting in CA due to amyloid accumulation increases thrombus development and raises the likelihood of stroke, which requires a risk assessment. Anticoagulation therapy tends to be protective, but further studies are necessary to determine their function as a prophylactic intervention to prevent stroke in these patients. The CS seems to have become more prevalent, however, this is likely due to improved imaging and/or a more thorough investigation rather than a real increase in prevalence. Corticosteroid reduces the burden of arrhythmias in cardiac sarcoidosis, but then the VT's mechanism is reentry due to scar formation in CS which is less susceptible to corticosteroid recovery.

Further studies are needed to explore the role of corticosteroids and other immunosuppressive drugs in 
reducing the burden of arrhythmias in cardiac sarcoidosis. Fatal arrhythmias usually lead to sudden death, which is not uncommon in both diseases, and appropriate ICD therapy to prevent these arrhythmias in cardiac amyloidosis has not provided any survival benefit and remains somewhat controversial. In contrast, it is particularly indicated in cardiac sarcoidosis. Sudden death is a disastrous outcome that has to be anticipated, and a better understanding of arrhythmia induced sudden death is required. The early use of ICD should be evaluated in more extensive studies that can help prevent sudden death due to arrhythmia. Ablation treatment only offers symptomatic relief from arrhythmias, so the recurrence risk is very high. Even with recent advancements in medicine, the overall prognosis remains rather dismal.

\section{Additional Information}

\section{Disclosures}

Conflicts of interest: In compliance with the ICMJE uniform disclosure form, all authors declare the following: Payment/services info: All authors have declared that no financial support was received from any organization for the submitted work. Financial relationships: All authors have declared that they have no financial relationships at present or within the previous three years with any organizations that might have an interest in the submitted work. Other relationships: All authors have declared that there are no other relationships or activities that could appear to have influenced the submitted work.

\section{References}

1. Gilstrap LG, Dominici F, Wang Y, et al.: Epidemiology of cardiac amyloidosis-associated heart failure hospitalizations among fee-for-service Medicare beneficiaries in the United States. Circ Heart Fail. 2019, 12:005407. 10.1161/CIRCHEARTFAILURE.118.005407

2. Yada H, Soejima K: Management of arrhythmias associated with cardiac sarcoidosis . Korean Circ J. 2019, 49:119-33. 10.4070/kcj.2018.0432

3. Mohty D, Damy T, Cosnay P, Echahidi N, Casset-Senon D, Virot P, Jaccard A: Cardiac amyloidosis: updates in diagnosis and management. Arch Cardiovasc Dis. 2013, 106:528-40. 10.1016/j.acvd.2013.06.051

4. McGrath DS, Goh N, Foley PJ, du Bois RM: Sarcoidosis: genes and microbes--soil or seed. Sarcoidosis Vasc Diffuse Lung Dis. 2001, 18:149-64.

5. John RM: Arrhythmias in cardiac amyloidosis. J Innov Card Rhythm Manag. 2018, 9:3051-7. 10.19102/icrm.2018.090301

6. Kandolin R, Lehtonen J, Airaksinen J, et al.: Cardiac sarcoidosis: epidemiology, characteristics, and outcome over 25 years in a nationwide study. Circulation. 2015, 131:624-32. 10.1161/CIRCULATIONAHA.114.011522

7. Desai R, Kakumani K, Fong HK, et al.: The burden of cardiac arrhythmias in sarcoidosis: a population-based inpatient analysis. Ann Transl Med. 2018, 6:330. 10.21037/atm.2018.07.33

8. Lin G, Dispenzieri A, Kyle R, Grogan M, Brady PA: Implantable cardioverter defibrillators in patients with cardiac amyloidosis. J Cardiovasc Electrophysiol. 2013, 24:793-8. 10.1111/jce.12123

9. Ballantyne B, Manian U, Sheyin O, Davey R, De S: Stroke risk and atrial mechanical dysfunction in cardiac amyloidosis. ESC Heart Fail. 2020, 7:705-7. 10.1002/ehf2.12602

10. Falk RH: Diagnosis and management of the cardiac amyloidoses. Circulation. 2005, 112:2047-60. 10.1161/CIRCULATIONAHA.104.489187

11. Brenner DA, Jain M, Pimentel DR, et al.: Human amyloidogenic light chains directly impair cardiomyocyte function through an increase in cellular oxidant stress. Circ Res. 2004, 94:1008-10. 10.1161/01.RES.0000126569.75419.74

12. Dorbala S, Vangala D, Bruyere J, et al.: Coronary microvascular dysfunction is related to abnormalities in myocardial structure and function in cardiac amyloidosis. JACC Heart Fail. 2014, 2:358-67. 10.1016/j.jchf.2014.03.009

13. Eriksson A, Eriksson P, Olofsson B-O, Thorneli L-E: The cardiac atrioventricular conduction system in familial amyloidosis with polyneuropathy. A clinicopathologic study of six cases from northern Sweden. Acta Pathol Microbiol Immunol Scand [A]. 1983, 91:343-9. 10.1111/j.1699-0463.1983.tb02765.x

14. Eriksson A, Eriksson P, Olofsson B-O, Thornell L-E: The sinoatrial node in familial amyloidosis with polyneuropathy. A clinico-pathological study of nine cases from northern Sweden. Virchows Arch (Pathol Anat). 1984, 402:239-46. 10.1007/BF00695078

15. Seward JB, Casaclang-Verzosa G: Infiltrative cardiovascular diseases: cardiomyopathies that look alike. J Am Coll Cardiol. 2010, 55:1769-79. 10.1016/j.jacc.2009.12.040

16. Kwong RY, Heydari B, Abbasi S, et al.: Characterization of cardiac amyloidosis by atrial late gadolinium enhancement using contrast-enhanced cardiac magnetic resonance imaging and correlation with left atrial conduit and contractile function. Am J Cardiol. 2015, 116:622-9. 10.1016/j.amjcard.2015.05.021

17. Barbhaiya CR, Kumar S, Baldinger SH, et al.: Electrophysiologic assessment of conduction abnormalities and atrial arrhythmias associated with amyloid cardiomyopathy. Heart Rhythm. 2016, 13:383-90. 10.1016/j.hrthm.2015.09.016

18. Hörnsten R, Pennlert J, Wiklund U, Lindqvist P, Jensen SM, Suhr OB: Heart complications in familial transthyretin amyloidosis: impact of age and gender. Amyloid. 2010, 17:63-8. 10.3109/13506129.2010.483114

19. Viles-Gonzalez JF, Pastori L, Fischer A, Wisnivesky JP, Goldman MG, Mehta D: Supraventricular arrhythmias in patients with cardiac sarcoidosis prevalence, predictors, and clinical implications. Chest. 2013, 143:108590. 10.1378/chest.11-3214

20. Cain MA, Metzl MD, Patel AR, et al.: Cardiac sarcoidosis detected by late gadolinium enhancement and prevalence of atrial arrhythmias. Am J Cardiol. 2014, 113:1556-60. 10.1016/j.amjcard.2014.01.434

21. Roberts WC, McAllister HA Jr, Ferrans VJ: Sarcoidosis of the heart. A clinicopathologic study of 35 necropsy patients (group I) and review of 78 previously described necropsy patients (group II). Am J Med. 1977, 63:86- 
108. 10.1016/0002-9343(77)90121-8

22. Feng D, Edwards WD, Oh JK, et al.: Intracardiac thrombosis and embolism in patients with cardiac amyloidosis. Circulation. 2007, 116:2420-6. 10.1161/CIRCULATIONAHA.107.697763

23. Feng D, Syed IS, Martinez M, et al.: Intracardiac thrombosis and anticoagulation therapy in cardiac amyloidosis. Circulation. 2009, 119:2490-7. 10.1161/CIRCULATIONAHA.108.785014

24. Russo D, Limite LR, Arcari L, Autore C, Musumeci MB: Predicting the unpredictable: how to score the risk of stroke in cardiac amyloidosis. J Am Coll Cardiol. 2019, 73:2910-1. 10.1016/j.jacc.2019.02.078

25. Dubrey SW, Cha K, Anderson J, et al.: The clinical features of immunoglobulin light-chain (AL) amyloidosis with heart involvement. QJM. 1998, 91:141-57. 10.1093/qjmed/91.2.141

26. Sayed RH, Rogers D, Khan F, et al.: A study of implanted cardiac rhythm recorders in advanced cardiac AL amyloidosis. Eur Heart J. 2015, 36:1098-105. 10.1093/eurheartj/ehu506

27. Mlcochova H, Saliba WI, Burkhardt DJ, et al.: Catheter ablation of ventricular fibrillation storm in patients with infiltrative amyloidosis of the heart. J Cardiovasc Electrophysiol. 2006, 17:426-30. 10.1111/j.15408167.2005.00321.x

28. Kumar S, Barbhaiya C, Nagashima K, et al.: Ventricular tachycardia in cardiac sarcoidosis: characterization of ventricular substrate and outcomes of catheter ablation. Circ Arrhythm Electrophysiol. 2015, 8:87-93. 10.1161/CIRCEP.114.002145

29. Naruse Y, Sekiguchi Y, Nogami A, et al.: Systematic treatment approach to ventricular tachycardia in cardiac sarcoidosis. Circ Arrhythm Electrophysiol. 2014, 7:407-13. 10.1161/CIRCEP.113.000734

30. Orini M, Graham AJ, Martinez-Naharro A, et al.: Noninvasive mapping of the electrophysiological substrate in cardiac amyloidosis and its relationship to structural abnormalities. J Am Heart Assoc. 2019, 8:012097. 10.1161/JAHA.119.012097

31. Reisinger J, Dubrey SW, Lavalley M, Skinner M, Falk RH: Electrophysiologic abnormalities in AL (primary) amyloidosis with cardiac involvement. J Am Coll Cardiol. 1997, 30:1046-51. 10.1016/s0735-1097(97)00267-2

32. Ridolfi RL, Bulkley BH, Hutchins GM: The conduction system in cardiac amyloidosis. Clinical and pathologic features of 23 patients. Am J Med. 1977, 62:677-86. 10.1016/0002-9343(77)90870-1

33. Nery PB, Beanlands RS, Nair GM, et al.: Atrioventricular block as the initial manifestation of cardiac sarcoidosis in middle-aged adults. J Cardiovasc Electrophysiol. 2014, 25:875-81. 10.1111/jce.12401

34. Sadek MM, Yung D, Birnie DH, Beanlands RS, Nery PB: Corticosteroid therapy for cardiac sarcoidosis: a systematic review. Can J Cardiol. 2013, 29:1034-41. 10.1016/j.cjca.2013.02.004

35. Padala SK, Peaslee S, Sidhu MS, Steckman DA, Judson MA: Impact of early initiation of corticosteroid therapy on cardiac function and rhythm in patients with cardiac sarcoidosis. Int J Cardiol. 2017, 227:565-70. 10.1016/j.ijcard.2016.10.101

36. Willner JM, Viles-Gonzalez JF, Coffey JO, Morgenthau AS, Mehta D: Catheter ablation of atrial arrhythmias in cardiac sarcoidosis. J Cardiovasc Electrophysiol. 2014, 25:958-63. 10.1111/jce.12424

37. Papageorgiou N, Providência R, Bronis K, et al.: Catheter ablation for ventricular tachycardia in patients with cardiac sarcoidosis: a systematic review. Europace. 2018, 20:682-91. 10.1111/jce.12424

38. Birnie DH, Sauer WH, Bogun F, et al.: HRS expert consensus statement on the diagnosis and management of arrhythmias associated with cardiac sarcoidosis. Heart Rhythm. 2014, 11:1305-23.

10.1016/j.hrthm.2014.03.043 